Pratiques d'écriture et champs professionnels. Petites fabriques d'auteur

\title{
Écrits de travail : sur la piste de l'auteur
}

Writing in a work context: on the writer's trail.

\section{Françoise Rouard}

\section{(2) OpenEdition \\ Journals}

Édition électronique

URL : http://journals.openedition.org/edc/2492

DOI : 10.4000/edc. 2492

ISSN : 2101-0366

Éditeur

Université Lille-3

\section{Édition imprimée}

Date de publication : 1 janvier 1995

Pagination : 41-54

ISSN : $1270-6841$

\section{Référence électronique}

Françoise Rouard, «Écrits de travail : sur la piste de l'auteur », Études de communication [En ligne], 16 | 1995, mis en ligne le 22 juin 2011, consulté le 02 mai 2019. URL : http://journals.openedition.org/ edc/2492 ; DOl : 10.4000/edc.2492

Ce document a été généré automatiquement le 2 mai 2019.

(c) Tous droits réservés 


\title{
Écrits de travail : sur la piste de l'auteur
}

Writing in a work context: on the writer's trail.

\author{
Françoise Rouard
}

« La vérité n'est pas toujours dans un puits. En somme, quant à ce qui regarde les notions qui nous intéressent de plus près, je crois qu'elle est invariablement à la surface. »

E.A.Poe

Double assassinat dans la rue Morgue

1 Le domaine des écrits au travail constitue un objet récent de recherche. Pour être plus précis, nous pourrions dire qu'il s'agit d'un objet de recherche en voie de constitution, à propos duquel de nombreux travaux empiriques seront encore nécessaires, avant que de véritables stabilisations conceptuelles soient possibles et productives. Que des lois communes les régissent, c'est ce que de nombreux travaux postulent par delà leur diversité. Qu'elles soient susceptibles de converger et d'être ordonnées pour définir un objet formalisable, voilà ce qui représente un nouveau continent à défricher.

2 N'est-il pas dès lors fort présomptueux de poser - comme la charrue avant les bœufs - la question de l'auteur dans ce territoire vague où n'existe aucune voie conceptuelle royale? N'est-ce pas céder, à la fois à la précipitation et à l'illusion d'une catégorie unitaire élaborée sous d'autres cieux et que l'on emploierait dans un cadre qui lui est étranger, voire rétif?

3 Les lignes qui vont suivre se veulent hypothétiques et heuristiques. Elles sont moins consacrées à l'auteur dans les écrits de travail qu'aux conditions de possibilité de la position de cette question. On l'aura compris : avant de poser la question, nous voulons savoir comment elle se pose, dans la double relation qui l'institue comme telle, au regard de nos représentations de la notion d'auteur d'une part, et au regard des éléments de savoir déjà élaborés dans le champ des écrits de travail, d'autre part. 
Cette phase de notre travail est, à l'évidence, critique et négative avant tout. Mais Definitio est negatio - disait Spinoza - elle est peut-être l'antichambre de futures élaborations positives.

\section{L'auteur: le paradigme dominant de l'art}

La notion d'auteur relève du territoire de l'art et plus particulièrement, à l'intérieur de ce dernier, de la région « littérature ». Il est nécessaire de s'y arrêter un instant pour en cerner les traits formels principaux et - grosso modo - le contour rationnel.

6 1.1. A la question «qu'est-ce qu'un auteur? » la réponse immédiate la plus fréquente est " celui qui écrit des romans ». Ce premier niveau d'approche révèle une " connaissance » de type spontané, réflexe pourrait-on dire, à condition de rattacher ce genre de réflexe à toute une série de déterminants qui le conditionne dans notre culture occidentale.

7 A la regarder de près, cette réponse n'est pas aussi superficielle qu'elle paraît : elle pose dans un même mouvement les catégories de sujet (celui), de produit du sujet (roman) et d'acte nécessaire (a écrit). Elle suppose en outre une connaissance du roman comme genre distinct d'écriture (différent de la poésie, de la science, de la théorie en général) assez vite qualifié d'" histoire ", de " fiction » dès qu'on demande des précisions à l'interlocuteur.

Elle pose enfin à l'intérieur de la définition qu'elle constitue une logique de l'attribution: est auteur, celui ou celle qui a écrit le produit que j'appelle roman. Le fait d'écrire est donc fondateur, mais seulement si l'on en reste à ce stade de définition générale donné par x lecteur général. Car si l'on précise la question sous la forme " quel est l'auteur de tel ou tel roman?" la réponse (qu'elle soit correcte ou erronée) s'orientera immanquablement vers un nom propre. Ce nom, c'est-à-dire la signature, constitue une deuxième modalité de l'attribution, mais cette fois nettement plus riche en termes d'indication : elle peut, en effet, renvoyer à une époque, à une école littéraire, à un style etc... Elle précise et différencie à la fois. Retenons ce point: l'attribution de la qualité d'auteur à un sujet relève spontanément de deux modalités opérant à des niveaux différents, le fait d'écrire et la signature.

9 1.2. Réintégrons à présent ces catégories dans l'univers des auteurs. Dans ce domaine, inutile de poser des questions. La multitude d'interviews publiés dans la presse, ou diffusés à la radio et à la télévision sont un matériau suffisant. Sans nous aventurer dans le domaine multiple des raisons de chacun, il est impossible de ne pas reconnaître au sein de ces discours l'usage massif de phrases du type : «J'ai écrit ce livre parce que je voulais dire ... » « j'ai éprouvé le besoin le désir/la nécessité... de décrire..., j’ai décidé d'écrire ce livre pour dire/témoigner/montrer... «j'ai voulu exprimer... ».

Les humoristes, on le sait, utilisent ces éléments jusqu'à la caricature. Mais si l'on est frappé par la pauvreté répétitive de ces phrases, elles n'en recèlent pas moins le même degré d'intérêt que la réponse naïve de notre lecteur décrit plus haut. Elles placent toutes, en effet, le centre de gravité de la notion d'auteur vers le " je » décrit comme le foyer central d'une volonté d'expression, d'une décision d'écrire. J'écris parce que je veux, je suis auteur, parce que dans mon intériorité propre je l'ai décidé. En écrivant, je m'en suis donné les moyens. En publiant tel livre, j'ai assumé dans le monde et ma décision et ma volonté. En ce sens, l'acte d'écrire est second, provoqué par l'acte de volition. La plupart des auteurs, quand ils discourent sur leurs livres articulent nécessairement une méta-physique de l'intention du sujet libre et tout puissant, origine 
dernière de leur expression et choc initial de l'acte d'écrire. Dans cet espace, la signature ne renvoie ni à un style, ni à une école, ni à un temps, mais bien à l'authentification de la décision préalable, c'est-à-dire à une prise de responsabilité. En signant, je fais plus que m'attribuer le produit de mon écriture, j'indique dans une aveuglante clarté que j'assume ma décision/volonté dans toute sa dimension et que mon moi, mon identité coïncide parfaitement avec elle. En bref, le « je » persiste et signe!

11 1.3. Il n'est pas question dans cet article d'esquisser une impossible théorie de la littérature. Par contre, dans notre tentative modeste d'éclaircissement de nos conceptions «spontanées» de l'auteur, il est nécessaire de souligner la remarquable inversion de valeur que produit le passage de lecteur à auteur en termes de représentation.

12 Dans sa définition de l'auteur, le lecteur valorise l'acte concret d'écrire et procède à une attribution première par le faire; le nom (la signature) est pour lui une attribution de spécification engageant des différenciations de style, de sujet, etc...

13 L'auteur quant à lui, bascule la priorité vers son moi, origine de volonté et de décision, sa signature en est l'authentification.

14 Même s'ils coexistent, ces deux points de vue ne sont pas équivalents. Ils relèvent, à l'évidence, de pratiques différentes mais le second - celui de l'auteur - est dominant, c'est lui qui « donne le ton ». La légitimité de cette domination peut être mise en question mais ce n'est pas l'objet de ce travail. Qu'il nous suffise de noter qu'elle s'enracine dans deux opinions qu'on peut selon le cas tenir pour des préjugés :

- L'autorité «naturelle » du discours de « celui qui fait », sur sa propre pratique ;

- l'adéquation du discours de l'auteur avec une représentation spontanée du sujet, dominante en Occident.

En termes pratiques, cette domination se révèle la plupart du temps dans le fait que les lecteurs reconnaissent volontiers que si l'auteur signe, c'est qu'il "prend ses responsabilités ». Cette attitude a sans aucun doute son fondement dans l'idée juridique " vulgaire » que signer c'est s'engager, mais dans la mesure où la constitution même de notre droit moderne est allée de pair avec celle de l'individu libre et responsable, ils rejoignent en douceur le terrain où l'auteur campe, fermement appuyé sur son « moi » décideur et volontaire.

16 Il (elle) a signé, c'est que c'est bien lui qui a écrit et en signant il prouve qu'il a écrit - ce que l'auteur ne cesse de clamer. C.Q.F.D.

\section{Idées claires et terrain vague}

17 Est-il possible d'appliquer, telle qu'elle nous est apparue, la notion d'auteur dans le domaine des écrits de travail ? Sont-ils susceptibles d'entrer pleinement sous les traits formels et rationnels qu'engage cette problématique de l'intentionnalité, du vouloir dire ${ }^{1}$ supposés de l'auteur dans nos représentations spontanées-dominantes?

18 2.1. Comparés au jardin «à la française » de nos conceptions littéraires multiséculaires, les écrits du travail font figure de jungle; c'est la "zone». Ce constat a déjà été clairement établi (Pène 1994, Rouard 1994): grisaille, éloignement de tout éclat stylistique, carac-tère procédural, utilitarisme, volonté opérationnelle, mise à l'écart de la subjectivité au profit évident des actes du travail et des objectifs de l'entreprise. En outre, la variété de leurs formes (mémos, notes de services, rapports d'intervention, évaluations, 
rapports de suivi, etc...) paraît relever plus de la diaspora que de la cohérence. Enfin, ils peuvent être signés ou non, leur « émetteur " n'en est pas forcément le rédacteur et le signataire ne coïncide pas obligatoirement avec le fabricant du texte ni avec son scripteur. Ce qui fait éclater la chaîne linéaire Volonté (intention) > Acte (écriture) > signature (responsabilité). La question de la signature est ici intrigante au plus haut point. Non signés, ces textes renvoient à un fonctionnement "objectif» de l'entité entreprise comme organisme. - C'est souvent le cas des notes de service ou des notes à l'attention du personnel: «A partir du 3 janvier, la cantine ouvrira à $11 \mathrm{~h} 45$ »; « un vendredi sur quatre les personnels pourront partir à 17 heures »... Les destinataires de ces notes ne peuvent que les attribuer par défaut au collectif anonyme de « la Direction » ou des «Ressources Humaines». Souvent même, ce sont ces collectifs qui servent de signature : dans ce cas, c'est à une fonction et non à un individu que renvoie l'acte de signer. Nous voici bien loin du sujet, de son intentionnalité expressive, et du «vouloir dire soi ».

2.2. Qu'en est-il quand le texte est individuellement signé par son rédacteur? Nous avons récemment examiné ce cas (Rouard 1994) mais avec un autre point de vue, dans une étude sur les rapports de maintenance.

De prime abord, nous semblons rentrer parfaitement dans la catégorie d'auteur telle que nous la manipulons spontanément. L'air de famille est évident! Nous avons les trois éléments indispensables à cette opération : le sujet, l'écrit, la signature. Seulement voilà, les airs de famille ne font pas les liens de parenté et la pure analyse morphologique révèle ses limites, quand on rapporte les traits formels qui la constituent aux configurations pratiques et idéologiques au sein desquelles ils fonctionnent.

Or l'écrit de travail se produit à l'intérieur d'un dispositif contraint. Le fait d'écrire est une obligation liée non seulement à l'exercice normal du métier mais également à la politique de l'entreprise en tant que personne morale. Dans le cas des écrits de maintenance, par exemple, il est clair que ces rapports d'intervention sont un élément de l'activité de l'expert au même titre que ses capacités à résoudre les pannes. Il est clair également qu'ils constituent un sous-ensemble, nécessaire à l'intérieur de la relation contractuelle existant entre l'entreprise et son client, sous-ensemble utilisé selon les cas de figure pour le suivi des situations, leur validation mutuelle, pour faire preuve dans l'hypothèse d'un litige ...

22 Face à cette norme de fer, l'écrit au travail est nécessairement un écrit pauvre en contenu subjectif : acte d'écrire obligé, dédié explicitement à la description des situations où priment l'exercice du métier et les intérêts de l'organisation ; sa signature est une prise de responsabilité au regard d'instances non individuelles, elle signe plus un professionnalisme qu'une subjectivité.

23 Nous voici bien loin de la galaxie rationnelle qui servait de socle à notre notion d'auteur, aux antipodes même ! C'est à l'intérieur de cette inversion axiale qu'il nous faut tenter de répondre à notre question : comment poser la question de l'auteur des écrits au travail ? 2.3. Au cours de notre approche des écrits de maintenance, nous avons émis plusieurs hypothèses applicables à un texte écrit par celui qui le signe. Rappelons les brièvement.

\section{a) Un discours contradictoire à dominance}

Le discours de l'écrit au travail ne ressort du sujet qu'en tant qu'il est le suppôt individuel d'une activité normée qui le dépasse. Ce discours est donc avant tout celui de ce suppôt et 
non celui de l'individu dans son intériorité. Le discours du suppôt s'articule dans une relation fondamentale et dominante avec les attentes et les objectifs de l'organisation comme être collectif. A ce stade, le rédacteur-signataire est un peu dans la position du philosophe platonicien, qui tend à proférer l'Être dans sa rectitude, et non lui même comme singularité secondaire (l'inverse constituant la position sophiste).

Ce discours est contradictoire en puissance dans la mesure où il doit nécessairement passer par un individu dont l'intérêt est soit de maintenir sa position de suppôt, soit d'en changer, parfois les deux.

Entre la notion d'individu et celle de suppôt de l'organisation, il n'y a pas identité, mais au contraire décalage. L'adéquation est un état transitoire et un équilibre instable. Car vouloir durer dans sa fonction, c'est vouloir se protéger, donc éventuellement ruser, mentir, dissimuler pour établir dans ses écrits un ordre de succès, ou à défaut de nonresponsabilité. Vouloir progresser, changer de poste, « réussir » comme on dit, c'est viser une autre position de suppôt, donc également ruser dans la position de suppôt actuel. Dans les deux cas, le risque est un compagnon de route.

Le décalage des intérêts entre l'organisation et l'individu conditionne le décalage individu/suppôt. Ce dernier s'exprime par la mise en place d'une stratégie individuelle discursive dont le champ est constitué par les étroites marges de manœuvre que laisse la domination ouverte du discours de l'organisation.

\section{b) L'individu (l'auteur) une notion indirecte et lacunaire}

Le terme de stratégie employé ici ne doit pas faire illusion. Ne pouvant s'exprimer qu'à l'intérieur du discours contraint de l'organisation, la stratégie discursive individuelle se déploie à la surface de ce dernier de façon lacunaire, dans les interstices qu'on lui laisse ou qu'elle prend parfois par irruption brève. Ceci nous amène à deux constatations :

- Chaque texte pris isolément est un manteau troué du point de vue de cette stratégie. Il y manque des pièces. Pour reconstituer le puzzle, il faudrait pour un même individu posséder l'ensemble des textes produits et signés pendant l'occupation d'un poste déterminé.

- Pour spécifier tel individu par rapport à tel autre, il faudrait disposer pour plusieurs individus, en même temps, de l'ensemble de leurs textes. Alors et seulement alors, une connaissance approchant véritablement la notion d'auteur serait possible. Par le repérage de tournures, de mots, bref par la mise en place d'indicateurs linguistiques stabilisés pour chacun, l'attribution serait véritablement possible.

Encore faut-il préciser que le croisement des styles avec une vision globale des stratégies ne livrerait qu'une infime partie des individualités en cause : il renvoie à eux de façon indirecte, il fait signe dans leur direction. La possibilité de l'opérer représente néanmoins une avancée significative pour construire la notion d'auteur dans les écrits de travail.

\section{L'auteur : éléments d'un savoir indiciaire}

31 Les obstacles, on l'a vu, sont nombreux sur notre route. Si repérer l'auteur, c'est repérer l'individu, alors nous sommes nécessairement conduits à une espèce d'enquête dont l'objet lui-même est une stratégie qui entretient un rapport indirect à l'expression indivi-duelle. 
Dans les écrits au travail, l'auteur est donc nécessairement ce qu'à la suite de C. Ginzburg (1989) nous pouvons appeler l'objet d'un savoir indiciaire. Quelques éléments méthodologiques s'imposent ici.

3.1. La situation de l'écrit au travail, où, par-delà la possession d'une rédaction signée, c'est l'individu lui-même qui se dérobe, trouve son répondant dans une situation bien connue: l'attribution de tableaux. Entre 1874 et 1876, Giovanni Morelli publia sous le pseudonyme de Lermolieff une série d'articles proposant une méthode d'attribution dans le domaine. Le problème était simple : les musées regorgent de tableaux inexactement attribués et l'entreprise est rendue très complexe par l'absence de signature, par le mauvais état de conservation voire parce que l'œuvre a été repeinte. Sa méthode prétendait non seulement distinguer un original d'une copie, mais permettre également de déterminer si, pour une « école » donnée le maître était ou non intervenu. La méthode de Morelli recommandait de délaisser les traits les plus apparents d'un tableau pour s'attacher à l'examen des détails les plus secondaires et les moins déterminés par l'école du peintre supposé, (lobes d'oreille, ongles, formes des doigts de mains et des pieds etc... ). C. Ginzburg note que la démarche de Morelli est à peu de chose près celle de Sherlock Holmes :

«En art, le connaisseur est comparable au détective qui découvre l'auteur du délit/ du tableau sur la base d'indices imperceptibles pour la plupart des gens »;

E. Wind (1963) écrit à propos de Morelli :

«(...) La psychologie moderne serait certainement du côté de Morelli : nos petits gestes inconscients révèlent davantage notre caractère que n'importe quel comportement formel, soigneusement préparé par nous. »

3.2. Le relais dans les sciences humaines est pris par Freud dans son célèbre essai de 1914 : « Le Moïse de Michel-Ange » où il écrit :

«Longtemps avant que je fusse à même d'entendre parler de la psychanalyse, j'appris qu'un amateur d'art de nationalité russe, Ivan Lermolieff (...) avait provoqué une véritable révolution (...) en construisant de nouvelles individualités artistiques (...). Il parvient à ce résultat en commençant par détourner le regard de l'impression d'ensemble ou des grands traits du tableau et en mettant en relief l'importance caractéristique des détails secondaires, des vétilles (...). Je crois que son procédé est étroitement apparenté à la technique de la psychanalyse médicale." (souligné par nous F.R.).

En quoi consiste ce lien de parenté ? Très clairement dans la mise en place d'une méthode d'analyse et d'interprétation fondée sur les écarts, sur le repérage et la mise en valeur de traces, de faits secondaires considérés comme révélateurs. Du lapsus freudien au lapsus pictural (celui du copieur qui « disjoncte » dans le tracé d'un ongle) la ligne est directe : les traces secondaires deviennent prépondérantes. Morelli, Conan Doyle, Freud constituent donc pour nous les emblèmes d'une démarche indiciaire à laquelle nous nous rattachons. Comment?

3.3. Nous formulons l'hypothèse d'une parenté forte entre notre objet et celui des trois auteurs cités plus haut.

Nous avons défini l'écrit au travail comme un discours à dominance, c'est-à-dire un discours où l'expression subjective doit nécessairement se couler dans le discours désincarné de l'organisation pour advenir, et pouvoir profiter de marges, d'interstices, d'écarts. Nous avons également établi que, de notre point de vue, ce discours de l'individu n'a rien d'un continuum mais se repère dans des traces infimes: flexions verbales, 
éléments de contexte, jeu de pronoms, choix de verbes etc..., c'est-à-dire dans une série d'« événements » discursifs mineurs ou marginaux survenant à la surface même du discours dominant et normé que porte le suppôt. L'individu (l'auteur) est donc, par hypothèse, parfaitement visible, " devant ", sous le regard même qui doit le saisir. Mais le saisir, non tel qu'on le supposait et où on l'attendait (entier et continu), mais épars, morcelé, lacunaire... Cette structure d'objet à dominance est également celle de l'objet de la psychanalyse : il n'y a pas de discours de l'inconscient en dehors du discours conscient du patient et de la domination du moi. Tout comme le travail de Morelli est de saisir au sein d'un discours pictural dominant des signes incontrôlés par l'auteur du tableau.

La différence avec notre propos réside sans doute, au stade actuel, dans la notion de contrôle. Le terme de stratégie que nous employons pour le moment, faute de mieux, traîne avec lui une idéologie de la conscience et de la maîtrise. Cette objection n'est pas irrecevable, mais au niveau de travail empirique où nous nous situons encore, elle n'est pas rédhibitoire. Car, dans les écrits au travail, cette conscience et ce contrôle jouent et sont largement efficaces. La catégorie de stratégie pourra, à un moment donné, jouer le rôle d'obstacle épistémologique pour aller plus avant, mais loin s'en faut, nous n'avons pas encore touché cette limite. Par ailleurs, la prudence avec laquelle il faut construire et manipuler les concepts de traces et d'indices représente à elle seule, à l'intérieur de ma méthodologie, un signal d'alarme bénéfique.

\section{Sur la piste de l'auteur}

Les écrits au travail sont un domaine rétif à la notion classique d'auteur. Prise dans son aspect irréductible de subjectivité coïncidant avec elle-même par des écrits qu'elle authentifie de sa signature, elle pose problème pour ce qui nous concerne.

Dans les écrits au travail, le discours est contraint, obligé : il est celui de l'organisation, de ses procédures, de ses objectifs et intérêts. La signature ne renvoie pas au sujet mais au suppôt de l'organisation et l'auteur au sens classique n'est pas dans cette prise de responsabilité mais - en tant qu'individu - dans la fuite, la défausse, la stratégie discursive rusée, enlacée au discours dominant.

Les écrits au travail sont une jungle, nous l'avons dit. La représentation dominante de la notion d'auteur fait des écrits un zoo bien ordonné : ici les réalistes, là les naturalistes, plus loin les épiques, encore plus loin ceux de la fiction pure etc... A partir d'une structure commune, les auteurs se classent en styles, écoles, époques... Dans la jungle, rien de tout cela : les bêtes apparaissent de façon fugace, fondues dans le paysage, faisant corps avec lui... laissant seulement les traces de leur passage, indices de leur trajet, de leur conformation, de leurs buts...

43 Chercher l'auteur ici, c'est au sens propre de ce mot, le traquer être sur sa piste. Pour ce faire, la méthode du savoir indiciaire nous paraît la plus appropriée aujourd'hui par-delà ses imperfections et son importante dimension intuitive. Elle nous a aidé à déplacer les questions et à les reformuler, et c'est à partir d'elle que nous pouvons proposer plusieurs axes de recherche qui nous semblent féconds:

- Dégager un corpus de textes d'un même individu au cours de l'occupation d'un poste et construire le profil de la stratégie.

- Croiser à postes équivalents les corpus de plusieurs individus pour tenter de repérer des régularités dans les stratégies mais aussi des différences de styles. 
- Comparer ces travaux avec des travaux équivalents menés sur des textes émanant de secteurs différents dans les entreprises.

Voilà ce qu'on pourrait appeler un beau programme de recherche. On le voit la piste de l'auteur est longue mais dans ce domaine comme dans d'autres on ne devient pas chasseur en un jour.

\section{BIBLIOGRAPHIE}

Boutet, J., (1995), Une linguistique de l'activité, Langues et Langage - Le Seuil.

Delcambre, P., (1990), Ecritures professionnelles et pouvoirs - dans pratiques d'écriture et champs professionnels. Bulletin du Certeic $\mathrm{n}^{\circ} 11$ - Université Charles-de-Gaulle, Lille 3.

Derrida, J., (1962), La voix et le phénomène - PUF, Paris.

Freud, S., (1985), L'inquiétante étrangeté et autres essais - Gallimard, Paris.

Gardin, B., (1989), « Machine à dessiner ou machine à écrire : La production collective d'une dénomination »- Langages $n^{\circ} 93$.

Ginzburg, C., (1988), Signes piste et traces - Le Débat, Le Seuil, Paris.

Husserl, E., (1961), Recherches logiques (Tome II) - 1901. PUF, Paris.

Pène, S., (1994), Analyse de postes, bilans d'entretien écriture de procédures, « un prêt à écrire » pour inscrire l'activité - Education. Permanente $\mathrm{n}^{\circ} 120$.

Rouard, F., (1994), L'individu et ses traces : « objectivité » et stratégie dans les écrits de la maintenance - Education. Permanente $\mathrm{n}^{\circ} 120$.

Wind, E., (1963), Art et anarchy - Edit. Faber, London.

\section{ANNEXES}

Voici deux exemples d'écrits au travail dans l'univers de la maintenance il s'agit d'un télex et d'un compte rendu d'intervention. Pour des raisons de confidentialité, ils sont publiés sans références possibles, aux personnes ni aux entreprises en cause.

\section{ANNEXE 1}

\begin{tabular}{|l|l|}
\hline Service & $\mathrm{xxx}$ \\
\hline Département & $\mathrm{xxx}$ \\
\hline Pays & $\mathrm{xxx}$ \\
\hline
\end{tabular}


A titre d'information, pour ceux qui font des visites préventives, un certain M. ... est depuis 3 semaines à ... en charge d'établir des normes d'installation des machines informatiques en DR, au niveau de l'environnement, énergie, climatisation, sécurité, etc. Le résultat devrait paraître d'ici 3 semaines, 1 mois...

(Il a par ailleurs envoyé aux DR un questionnaire, différent du nôtre quant au contenu, mais qui a croisé, et parfois créé la confusion avec notre questionnement de visite).

Le 18/4193, à 14 h, T me signale qu'il y a une alerte noire à l'agence entreprise (c'est moi en effet qui ai participé le plus à la mise en service de l'établissement).

J'essaie de joindre par téléphone les ICL sur le site. Après leur recherche dans les étages, M. P me signale qu'il vient de rebooter tous les serveurs et qu'il demande aux utilisateurs de réinitialiser leur STB.

Avant, les impressions marchaient très mal, l'accès à la bureautique et aux sites distants étaient aléatoires : il insiste pour que L.B. intervienne.

Je joins L.B. et lui signale que je vais à l'agence MM, je la tiendrai au courant sur place et j'emmène le Sniffer.

Avec les bouchons (grève des transports en commun annoncée le matin), j'arrive sur le site à $16 \mathrm{~h}$ 15. Dans le bureau des ICL, tout a l'air de bien fonctionner sur leur STB. J'installe le Sniffer et on constate rien d'anorrnal (peu de collisions, 0 \% de bad FRS, quelques Bong Ack Clock de temps à autre). Avec des utilisateurs, nous testons les connexions qui ne fonctionnaient pas, 57M Noisy n'a pas marché une fois, mais ensuite cela a été. Nous réussissons également à imprimer au grand soulagement des utilisateurs. Le test R24 sur les 3 silos a fonctionné. L.B. me rappelle pour me demander ce qu'il en est. Je lui dis que tout est bon pour le moment et il se produit un But 21lD sur le Silos jaune (machine complètement bloquée). Des utilisateurs m'expliquent ce qu'ils avaient fait juste avant sur des STB jaunes : Connexion à 57M Chanzy réussie, connexion à Sophie réussie, puis connexion à 57M Chanzy échouée. $\mathrm{M}$. $\mathrm{P}$ et d'autres utilisateurs sont mécontents car leur impression sur une imprimante drivée par ce Silos est inachevée.

Après reboot du Silos, je demande à M. $\mathrm{P}$ de relancer sa demande d'impression (55 pages environ) de sa STB verte après qu'il l'ait rebootée. Tout se passe bien au début, d'autres utilisateurs utilisent le Silos jaune pour se connecter aux sites distants, mais avant que l'impression soit terminée, je m'aperçois que le Silos est de nouveau bloqué (il n'y a pas de But 21lD, Ctrl-Esc ne donne rien). Grand désarroi des utilisateurs.

Les imprimantes utilisées ici sont des Laser2, chaque STB voit une imprimante avec 3 files d'attente. L.B. ne souhaite pas rebooter le Silos jaune car elle va revenir le lendemain avec des disquettes.

Nous demandons à M. $\mathrm{P}$ de relancer son impression à partir d'une autre STB verte, mais placée à un autre endroit, mais qui utilise une imprimante drivée par le Silos vert. Tout se passe bien au début, vers la fin, nous faisons quelques tentatives de connexion sur des sites distants et le Silos vert se bloque.

Après reboot de ce Silos, nous allions relancer à nouveau l'essai d'impression sans faire du tout de connexions sur sites distants lorsque le courant à 19 h 15 s'est interrompu, coupant ainsi toutes les STB et imprimantes sous tension. Les onduleurs de la salle des serveurs ont pris la main et nous avons alors arrêté proprement tous les serveurs sauf le Silos jaune.

Fin de la journée.

Pierre, rapport d'intervention 


\title{
NOTES
}

1. Au plan philosophique, cette problématique a été développée jusque dans les ultimes conséquences par E. Husserl. Voir en particulier, Recherches logiques (T. II), 1901, Paris Puf, 1961. Voir également les pénétrantes analyses de J. Derrida in La voix et le phénomène, Paris Puf, 1962.

\section{RÉSUMÉS}

L'article s'interroge sur l'usage possible de la notion d'auteur pour analyser les écrits produits en contexte de travail. Le cadre contraignant de l'organisation qui exige l'écriture amène à analyser d'abord celui qui écrit comme le suppôt de cette organisation et non comme un auteur que l'usage définit comme un individu assumant sa décision et sa volonté d'écrire. Néanmoins on peut vouloir analyser les traces de l'individualité de l'écrivant. Il s'agira alors de s'inspirer des méthodes d'attribution des tableaux: l'individualité est lisible en surface,mais l'auteur n'est présent que de manière lacunaire; il se repère à des détails dans les interstices que l'ordonnancement organisationnel de l'écrit lui laisse ouverts. Fr. Rouard propose en conclusion un programme de travail qui permettrait une description des individualités dans l'écriture en contexte de travail.

The article investigates if it is productive to use the concept of « author » to analyse the writings produced within a work context. The restricting frame of the organisation requiring the worker to write leads first to describe him as a tool operating at a defined place in that organisation, rather than as an "author", in the sense of a free human being able to assume his own decision, and will, to write. Nethertheless, it is relevant to focus on an analysis of the identification marks to the worker who writes, «l'écrivant». One will then tranfer the methodology used for identifying the authors of painted masterpieces : the individuality can be identified reading the surface of the document, but the author is only intemittently present. The researcher spots him by interpreting details hidden inside instertices wich the organisational framing of the written product left open. The author suggests, as a conclusion, a methodology to describe the individuality of writing workers.

\section{INDEX}

Keywords : professional writing, author, signature, speech

Mots-clés : écriture professionnelle, auteur, signature, discours

\author{
AUTEUR \\ FRANÇOISE ROUARD \\ Françoise Rouard, C.N.A.M., GERICOfr
}

